\title{
Electrochemical Raman spectroscopy of carbon nanotube energy transfer complexes
}

\author{
Benjamin Hatting*, Friederike Ernst, Stephanie Reich
}

Institut für Experimentalphysik, Freie Universität Berlin, 14195 Berlin, Germany

Received XXXX, revised XXXX, accepted XXXX

Published online XXXX

Key words: carbon nanotube, Raman, energy tranfer

*Corresponding author: e-mail benjamin.hatting@fu-berlin.de

We present electrochemical Raman measurements on energy transfer complexes formed by combining singlewalled carbon nanotubes and dye molecules. The presence of the dye molecules in the proximity of the nanotubes impacts the nanotubes' charging behavior, leading to changes in the position of the charge neutrality point and the maximum achievable doping strength. We compare these results to spectra acquired in solution and determine the corresponding Fermi energy shifts, which are found to be similar in the energy transfer complexes and a reference sample. Our results have implications for the photoluminescence enhancement/quenching previously observed in these energy transfer complexes.

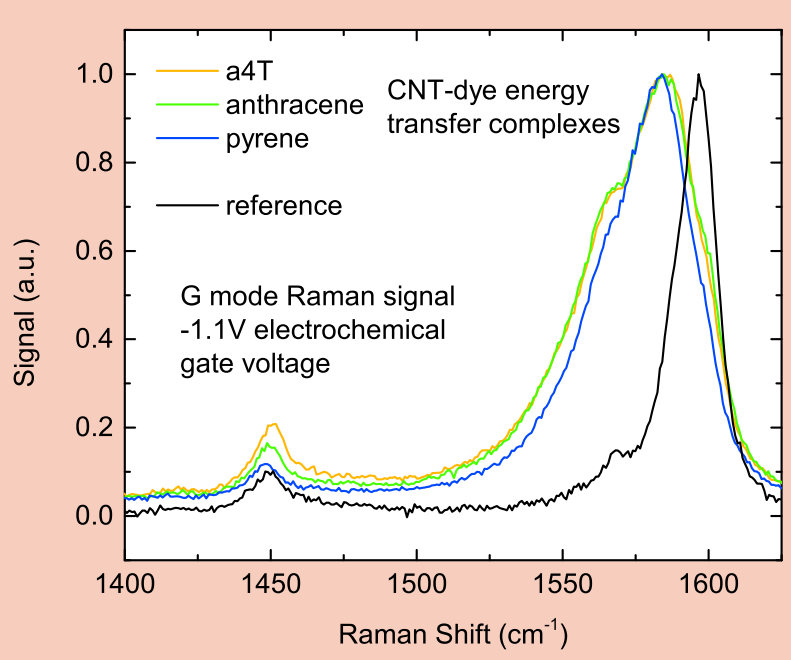

Electrochemical G-mode Raman spectra at a gate voltage of $-1.1 \mathrm{~V}$ showing much larger doping strength for the reference sample than for the energy transfer complexes, as seen in the higher-frequency and narrower peak.
1 Introduction Single-walled carbon nanotubes have attracted enormous research interest because of their varied optical properties [1], excellent electronic transport characteristics [2], and potential biomedical applications[3]. Semiconducting nanotubes emit light at near-infrared wavelengths, in the biomedically relevant transparency window of tissue[4].

Nanotubes have low intrinsic absorption strengths and different chiralities require different excitation energies, which hinders imaging application. Carbon nanotube en- ergy transfer complexes have recently been developed[510] to overcome these shortcomings. In these complexes, a dye featuring a higher intrinsic absorption strength is brought into proximity of the nanotube. After optical excitation of the dye, the excitation is transferred into the nanotube, and the nanotube emits light in the near infrared. The energy transfer efficiency reaches values that are very close to unity, meaning that almost every excitation created in the dye is transferred into the nanotube[5,7,9]. 
Spectroelectrochemical Raman measurements of carbon nanotubes have been used to investigate the electronphonon coupling and the charge states of nanotubes in solution and on substrates. The metallic LO component of the G-modelineshape is very sensitive to the Fermi energy, which can be changed by applying a gate voltage. The metallic LO peak exhibits a Kohn anomaly, which leads to a downshift of the peak compared to semiconducting tubes[11-17]. Furthermore, in metallic tubes there is an additional phonon relaxation channel consisting of electronic excitations between the linear bands crossing at the Fermi energy[18]. This additional phonon relaxation channel leads to a broader Raman peak. Both the Kohn anomaly and the phonon relaxation channel are only in effect at intrinsic Fermi energies and are lifted when the Fermi energy is shifted by half the phonon energy in either direction.

In this paper we conduct in-situ spectro-electrochemical Raman measurements on carbon nanotube energy transfer complexes formed using three different dye molecules and compare their behavior to that of a reference sample. We find that the energy transfer complexes exhibit different electrochemical charging characteristics such as a lower gating efficiency and shifted voltage position of the charge neutrality point. We also investigate the Fermi energy of complexes in solution and find that charge transfer between the constituents cannot explain the photoluminescence quenching observed previously for some of these complexes.

2 Methods and Samples Raman spectra were acquired on a Horiba XploRa spectrometer with $638 \mathrm{~nm}$ laser excitation. To prepare the energy transfer complexes, the hydrophobic dyes are introduced into the micelles and onto the tubes via micelle swelling as described in Ref. [6]. Consequently, there are no free dye molecules in the solution.We investigate three energy transfer complexes containing anthracene, pyrene, and $\alpha$-quarterthiophene(a4T), formed with PLV single-walled carbon nanotubes. For the electrochemical Raman measurements, the samples were dropcast from solution onto a gold-coated wafer to form the working electrode. Trimethylpropylammonium bis(trifluoromethanesulfonyl)imide was used as the electrolyte. The electrochemical cell was completed by a $\mathrm{Ag} / \mathrm{AgCl}$ reference electrode and a $\mathrm{Ag}$ wire counter electrode. Gate voltages between $-1.1 \mathrm{~V}$ and $+1.1 \mathrm{~V}$ were applied in steps of $0.1 \mathrm{~V}$ using a homemade voltage source.

3 Results and Discussion Figure 1 shows G-mode Raman spectra of the anthracene-carbon nanotube energy transfer complex acquired while sweeping the gate voltage from $0 \mathrm{~V}$ to $+1.1 \mathrm{~V}$. Spectra were also acquired at negative gate voltages. With increasing gate voltage, the metallic LO peak initially located at $1552 \mathrm{~cm}^{-1}$ upshifts and narrows in accordance with established results on the Kohn anomaly and the additional phonon relaxation channel, which are lifted and blocked at non-intrinsic Fermi ener- gies $[15,18]$. At the same time, the integrated peak intensity decreases. At larger gate voltages the semiconducting LO peak initially located at $1588 \mathrm{~cm}^{-1}$ additionally up-shifts to higher frequencies.

We fitted the electrochemical Raman spectra with three peaks for the G-mode region and track the peak position and width (full width at half maximum) of the lowestfrequency fit component, which corresponds to the metallic LO peak. The same type of measurement and analysis was performed for the pyrene-carbon nanotube and a4Tcarbon nanotube energy transfer complexes and the reference sample. Figure 2 shows the peak position and width as a function of gate voltage for all four samples.

Comparing the behavior of the three energy transfer complexes and the reference sample, we can make several observations: Firstly, the gate voltage at which the metallic LO peak exhibits the maximum width and minimum peak position is different for the different samples. This can be attributed to doping of the nanotubes by the dye molecules, which has to be countered by a certain gate voltage to achieve charge neutrality. The corresponding voltages are $+0.1 \mathrm{~V}$ for anthracene and $-0.1 \mathrm{~V}$ for pyrene and a4T. However, the spectrum of the reference sample also does not exhibit maximum broadening and minimum peak position at $0 \mathrm{~V}$, but instead at $+0.1 \mathrm{~V}$.

Secondly, the rate of change of the width and position of the metallic LO peak, i.e. the gate efficiency, is slightly smaller for the anthracene and pyrene samples than the a4T and the reference samples. This means that is harder to induce a given charge density in the nanotubes in these samples, presumably due to the presence of the dye molecules in immediate proximity to the nanotubes. To quantify the gate efficiency, we observe the difference in gate voltage between the two points where the peak width reaches half its maximum value (e.g. $+0.6 \mathrm{~V}-(-0.4 \mathrm{~V})=1 \mathrm{~V}$ for anthracene). From theory we know that this corresponds to a change in the Fermi energy equal to the phonon energy, $200 \mathrm{meV}[12,16,17]$. We thus arrive at a gating efficiency of $0.20 \mathrm{eV} / \mathrm{V}$ for anthracene. The gate efficiency is $0.20 \mathrm{eV} / \mathrm{V}$ for pyrene and $0.25 \mathrm{eV} / \mathrm{V}$ for a $4 \mathrm{~T}$ and the reference sample.

Thirdly, the most pronounced difference is in the minimum width and and maximum peak position of the metallic LO peak obtained at high gate voltages. Here the values for the reference sample indicate significantly stronger doping than for the energy transfer complexes, leading to a lower peak width and higher peak frequency. This observation can be hypothesized to be due to the presence of the dye molecules between the electric double layer and the nanotube, which reduces the maximum capacitance of the nanotube-electrolyte interface. This leads to lower maximum charge densities. The maximum doping in the reference sample is greater than in all three energy transfer complexes, while the behavior among the energy transfer complexes is fairly similar. Specifically, the peak width and position at $-1.1 \mathrm{~V}$ are $16 \mathrm{~cm}^{-1}$ and $1594 \mathrm{~cm}^{-1}$, respec- 


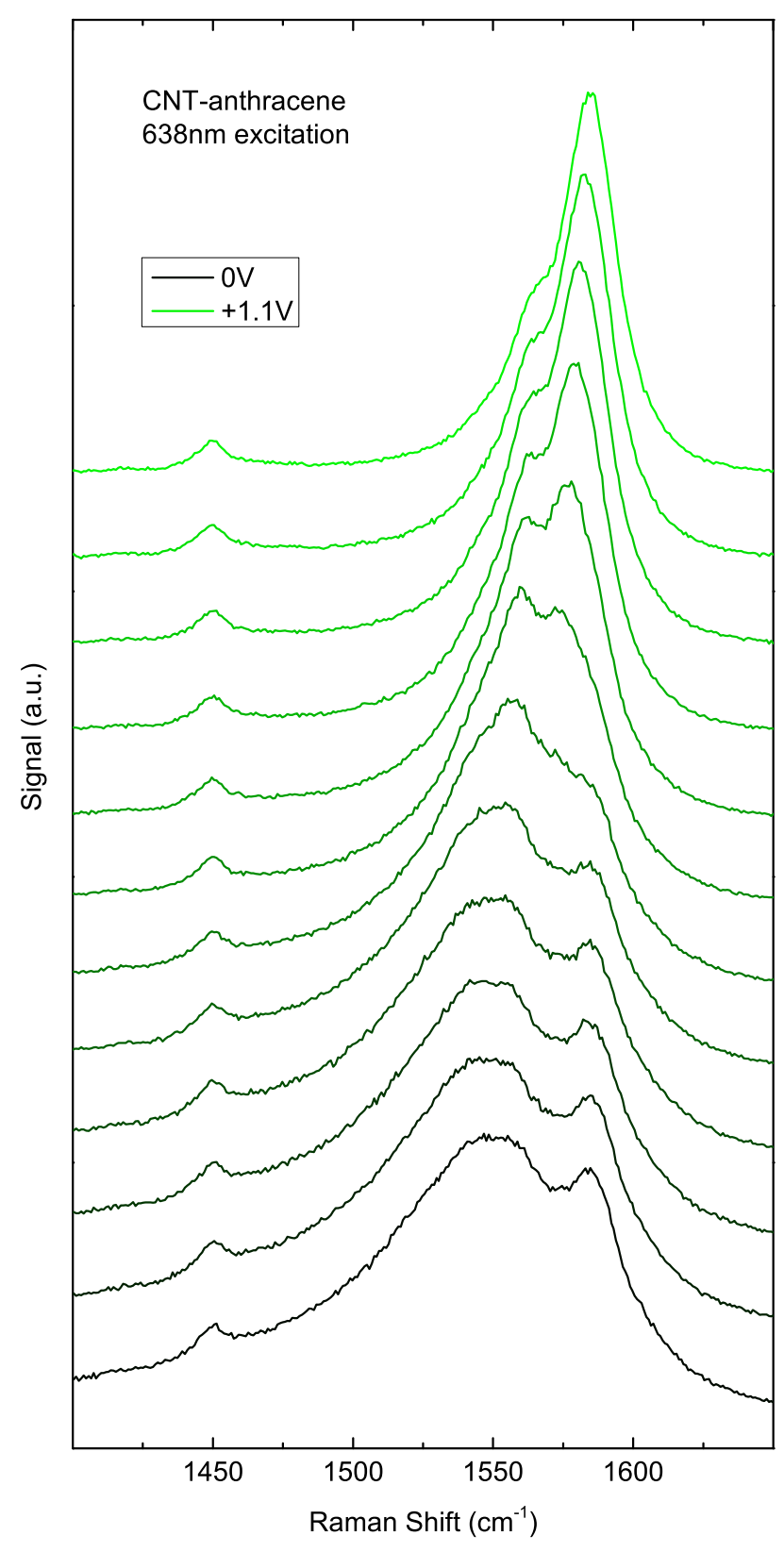

Figure 1 Electrochemical Raman spectra of the anthracenecarbon nanotube energy transfer complex acquired at gate voltages from $0 \mathrm{~V}$ to $+1.1 \mathrm{~V}$ with $638 \mathrm{~nm}$ laser excitation.

tively, compared to $32 \mathrm{~cm}^{-1}$ and $1579 \mathrm{~cm}^{-1}$ for anthracene. A comparison of the spectra at $-1.1 \mathrm{~V}$ is shown in Fig. 3b.

It is interesting to note that the maximum peak width of $80 \mathrm{~cm}^{-1}$ and the minimum peak position of $1550 \mathrm{~cm}^{-1}$ of the metallic LO peak obtained at the charge neutrality point is very similar in all the samples, see Fig. 3a. This proves that the dye molecules do not introduce significant Fermi energy inhomogeneity in the tubes, as could have arisen from uneven coverage of the tubes. Fermi energy inhomo-

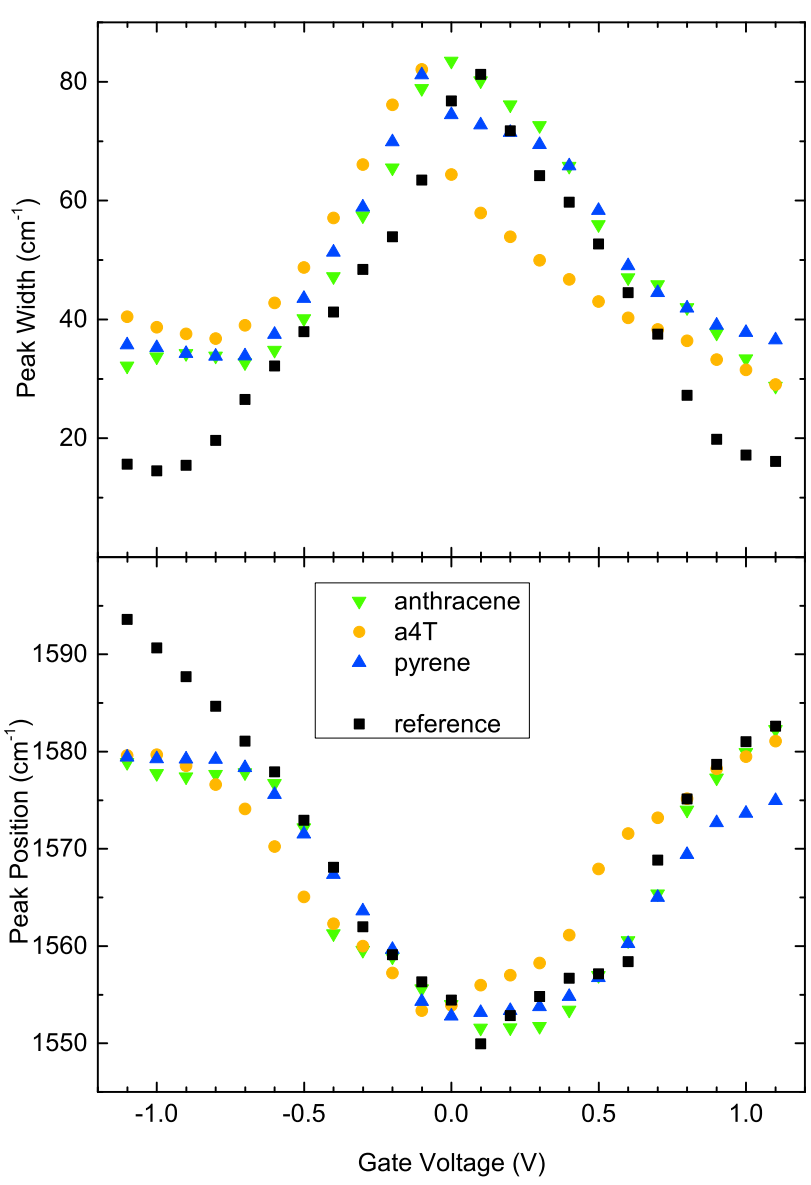

Figure 2 Peak width (full width at half maximum)and peak position of the metallic LO peak extracted from the fitted electrochemical Raman spectra as a function of gate voltage.

geneity would have manifested itself as a lower maximum peak width and higher minimum peak position because at a single gate voltage, not all the tubes would have been at charge neutrality.

Photoluminescence measurements on the carbon nanotube energy transfer complexes are typically carried out in solution. We therefore also compare Raman spectra of our samples acquired in solution to the electrochemical Raman spectra in order to assess the charge state of the nanotubes in solution, and in particular if the tubes are charged enough to quench their luminescence.

The G-mode Raman spectra in solution, shown in Fig. 3c, match the electrochemical Raman spectra taken at gate voltages of $-0.3 \mathrm{~V}$ for anthracene, $-0.4 \mathrm{~V}$ for pyrene and $\mathrm{a} 4 \mathrm{~T}$, and $-0.2 \mathrm{~V}$ for the reference sample. We take the voltage difference between these values and the respective voltages of the charge neutrality point and convert them to Fermi energy shifts using the gate efficiencies determined earlier in this paper. This calculation gives Fermi energy shifts of $80 \mathrm{meV}$ for anthracene and pyrene and $75 \mathrm{meV}$ 


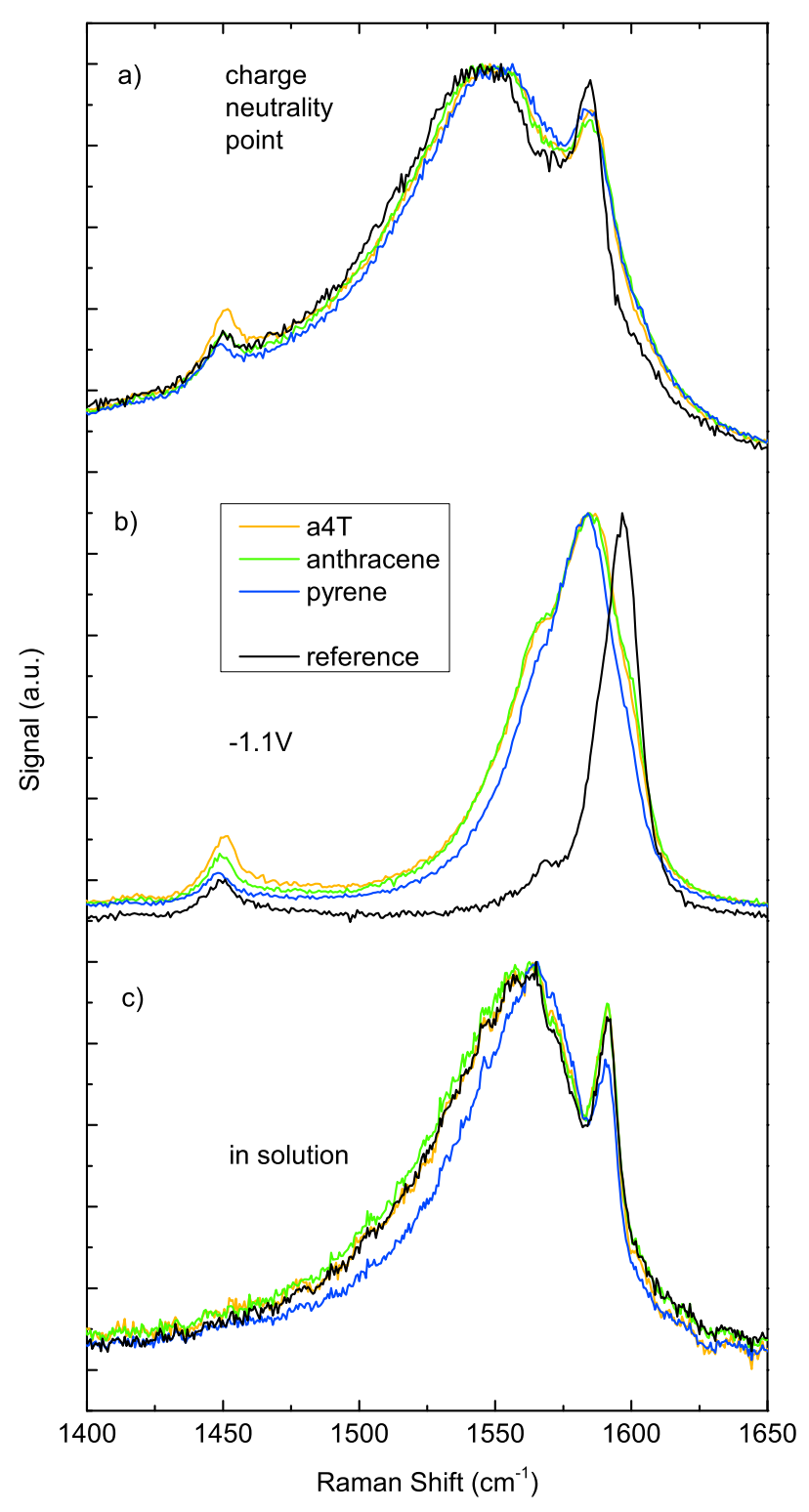

Figure 3 a) Spectra at charge neutrality showing a similar lineshape in all the samples. b) Spectra at $-1.1 \mathrm{~V}$ showing much larger doping strength for the reference sample than for the energy transfer complexes. c) Spectra in solution, from which the Fermi energy shifts were determined.

for a4T and the reference sample. These values are for the metallic tubes that contribute to the metallic LO peak.

Our measurements in this paper probe several metallic chiralities that contribute to the metallic LO peak in the spectrum together. By contrast the measurements in Ref. [6] probe various single semiconducting chiralities. It was found that a4T enhances the photoluminescence for all chiralities, by up to $100 \%$ for $(11,1)$ tubes. Anthracene quenches the photoluminescence of all chiralties by around $20 \%$, except for the $(11,1)$ chirality, which gives about $90 \%$ enhancement. Pyrene quenches the photoluminescence for all chiralities, by $30 \%$ on average. We do not focus on the Raman signal of semiconducting tubes in this work because their G-mode peak position and width is affected much less by changing the Fermi energy than the G-mode of metallic tubes.

In our measurements we found the spectra in solution to correspond to electrochemical potentials at most $0.4 \mathrm{~V}$ below the charge neutrality point. Assuming a charging efficiency of $1 \mathrm{eV} / \mathrm{V}$ for semiconducting tubes inside the band gap, these values would not be sufficient to shift the Fermi energy into the valence band, at which point quenching was observed in the absorption in Ref.[19].

Regardless of whether the Fermi energy shifts would be strong enough to quench the absorption or luminescence, since the Fermi energy shifts are very similar to the reference sample for all three energy transfer complexes, charge transfer cannot be the explanation for the photoluminescence quenching and enhancement observed in Ref. [6]. The photoluminescence quenching can be atributed to a reduced radiative life time in the energy transfer complexes. The precise mechanism of the radiative life time reduction was not determined. A possible candidate is the extrinsic local modulation of the Fermi energy through the adsorbed dye molecules leading to increased scattering.This local modulation of the Fermi energy is not accesible in our expermiments, which probe the average Fermi energy in an ensemble of nanotubes.

4 Conclusion To summarize, we performed electrochemical Raman measurements on the carbon nanotube energy transfer complexes. We found that the presence of the dyes influences the charging behavior of the nanotubes, as manifested in different positions of the charge neutrality point and reduced maximum doping strength. Furthermore, we examined the Raman spectra of the same complexes in solution and found that the Fermi energy has very similar values in all samples. This rules out charge transfer as the explanation for the different photoluminescence enhancement/quenching behavior of the different samples.

Acknowledgements S.R. acknowledges funding by the ERC (Grant No. 210642).

\section{References}

[1] K. Liu, J. Deslippe, F. Xiao, R. B. Capaz, X. Hong, S. Aloni, A. Zettl, W. Wang, X. Bai, S. G. Louie, E. Wang, and F. Wang, Nature Nanotechnology 7(5), 325-329 (2012).

[2] H. Park, A. Afzali, S. J. Han, G. S. Tulevski, A. D. Franklin, J. Tersoff, J. B. Hannon, and W. Haensch, Nature Nanotechnology 7(12), 787-791 (2012).

[3] A. L. Antaris, J. T. Robinson, O. K. Yaghi, G. Hong, S. Diao, R. Luong, and H. Dai, ACS Nano 7(4), 3644-3652 (2013).

[4] N. W. S. Kam, M. O'Connell, J. A. Wisdom, and H. Dai, Proc. Natl. Acad. Sci. U. S. A. 102(33), 11600-11605 (2005). 
[5] F. Ernst, T. Heek, A. Setaro, R. Haag, and S. Reich, Adv. Funct. Mater. 22(18), 3921-3926 (2012).

[6] F. Ernst, S. Heeg, T. Heek, A. Setaro, R. Haag, and S. Reich, physica status solidi (RRL) Rapid Research Letters 7(8), 546-549 (2013).

[7] F. Ernst, T. Heek, A. Setaro, R. Haag, and S. Reich, Appl. Phys. Lett. 102(23), - (2013).

[8] C. Roquelet, J. S. Lauret, V. Alain-Rizzo, C. Voisin, R. Fleurier, M. Delarue, D. Garrot, A. Loiseau, P. Roussignol, J. A. Delaire, and E. Deleporte, ChemPhysChem 11(8), 1667-1672 (2010).

[9] C. Roquelet, D. Garrot, J. S. Lauret, C. Voisin, V. AlainRizzo, P. Roussignol, J. A. Delaire, and E. Deleporte, Appl. Phys. Lett. 97(14), - (2010).

[10] F. Vialla, C. Roquelet, B. Langlois, G. Delport, S. M. Santos, E. Deleporte, P. Roussignol, C. Delalande, C. Voisin, and J. S. Lauret, Phys. Rev. Lett. 111(13), 137402- (2013).

[11] O. Dubay, G. Kresse, and H. Kuzmany, Phys. Rev. Lett. 88(23), 235506 (2002).

[12] K. Ishikawa and T. Ando, J. Phys. Soc. Jpn. 75(8), 084713 (2006).

[13] M. Lazzeri, S. Piscanec, F. Mauri, A. C. Ferrari, and J. Robertson, Phys. Rev. B 73(15), 155426 (2006).

[14] S. Piscanec, M. Lazzeri, J. Robertson, A. C. Ferrari, and F. Mauri, Phys.Rev. B 75(3), 035427 (2007).

[15] K. T. Nguyen, A. Gaur, and M. Shim, Phys. Rev. Lett. 98(14), 145504 (2007).

[16] N. Caudal, A. M. Saitta, M. Lazzeri, and F. Mauri, Phys. Rev. B 75(11), 115423 (2007).

[17] T. Ando, J. Phys. Soc. Jpn. 77(1), 014707 (2008).

[18] Y. Wu, J. Maultzsch, E. Knoesel, B. Chandra, M. Y. Huang, M. Y. Sfeir, L. E. Brus, J. Hone, and T. F. Heinz, Phys. Rev. Lett. 99(2), 027402 (2007).

[19] D. Paolucci, M. M. Franco, M. Iurlo, M. Marcaccio, M. Prato, F. Zerbetto, A. Pnicaud, and F. Paolucci, J. Am. Chem. Soc. 130(23), 7393-7399 (2008). 